\title{
Sin ley ni organismo técnico: la institucionalidad de la gestión de riesgo de desastres en la respuesta al terremoto de 2016 en Ecuador*
}

\author{
Hugo Cahueñas Muñoz**
}

\section{RESUMEN}

El artículo estudia el marco jurídico ecuatoriano relativo a la gestión de riesgos de desastres en dos momentos. Primero, se describe el marco jurídico que existía previo al terremoto del 16 de abril de 2016 (16A); y, en segundo lugar, se analiza el marco jurídico generado a partir de decretos expedidos como respuesta al terremoto. A base de este análisis se concluye que las acciones de respuesta ante el $16 \mathrm{~A}$ no se adecuaron plenamente al marco jurídico existente; al contrario, se presentó una sobreposición de funciones e instituciones. En consecuencia, teniendo presente el mandato del poder constituyente y la obligación estatal de proteger a las personas, las colectividades y la naturaleza de los efectos negativos de los desastres, el Ecuador debe adoptar una ley de gestión de riesgos que adecue la dispersión de normas e instituciones, colme las lagunas jurídicas, y solucione la inconstitucionalidad por la no adecuación legislativa.

PALABRAS ClAVE: desastres, terremoto, gestión de riesgo, estado de excepción, declaración de emergencia, respuesta a desastres, inconstitucionalidad, no adecuación legislativa.

\section{ABSTRACT}

The article studies the Ecuadorian legal framework related to disaster risk management in two moments. First, it describes the legal framework that existed prior to the earthquake of April 16, 2016 (16A); and, secondly, the legal framework generated from decrees issued in response to the earthquake is analyzed. Based on this analysis, it is concluded that the response actions to 16A were not fully adapted to the existing legal framework, on the contrary, an over position of functions and institutions was presented. Consequently, bearing in mind the mandate of the constituent power and the state obligation to protect individuals, communities and the

\footnotetext{
* La asistencia de investigación brindada por Angie Agama y Erick Guapizaca, estudiantes de pregrado de la Universidad San Francisco de Quito, constituyó un aporte básico en las etapas redacción y revisión.

** Docente del Colegio de Jurisprudencia de la Universidad San Francisco de Quito.
} 
nature of the negative effects of disasters, Ecuador must adopt a risk management law that adapts the dispersion of standards and institutions, fill the legal gaps, and solve the unconstitutionality due to the lack of legislative adaptation.

KEYwORDs: Disasters, earthquake, risk management, state of emergency, declaration of emergency, response to disasters, unconstitutionality, non legislative adaptation.

\section{INTRODUCCIÓN}

$\mathrm{U}$

n terremoto de 7,8 grados en la escala de Richter se registró en la región Costa del Ecuador el 16 de abril de 2016 (en adelante 16A), generando graves consecuencias humanitarias, entre las cuales se cuantifican: 663 personas fallecidas, 6.274 personas heridas, 113 personas rescatadas con vida, y cerca de treinta mil personas albergadas. ${ }^{1}$

Con el objetivo de gestionar los riesgos y consecuencias humanitarias de los desastres, el Marco de Sendai para la Reducción del Riesgo de Desastres 2015-2030 incluye como una de sus prioridades fortalecer la gobernanza del riesgo de desastres para gestionar el riesgo, para lo cual "es necesario contar con claros objetivos, planes, competencia, directrices y coordinación en los sectores y entre ellos". ${ }^{2}$ En la respuesta al terremoto del 16A se evidenció una débil gobernanza de la gestión de riesgos; por ejemplo, a las setenta y dos horas de ocurrido el terremoto, el Presidente de la República designó a varios "ministros para que asuman el control de la respuesta en cada uno de los cantones afectados". 3

Se debe tener presente que la gestión de riesgo de desastres, según la Estrategia Internacional para la Reducción de Riesgo de Desastres de las Naciones Unidas (UNISDR), es "el proceso sistemático de utilizar directrices administrativas, organizaciones,

1. Secretaría de Gestión de Riesgos, "Informe de Situación No. 71, 19/05/2016 (20h30)". Disponible en 〈http://www.gestionderiesgos.gob.ec/wp-content/uploads/downloads/2016/05/INFORME-n71-SISMO-78-20302.pdf $\rangle$. Consulta: 7 de junio de 2017.

2. Asamblea General de Naciones Unidas, Resolución A/RES/69/283: Marco de Sendai para la Reducción del Riesgo de Desastres 2015-2030, párr. 26.

3. Secretaría de Gestión de Riesgos, "Informe de Situación No. 65, 16/05/2016". Disponible en 〈http:// www.gestionderiesgos.gob.ec/wp-content/uploads/downloads/2016/05/Informe-de-situación-n ${ }^{\circ} 65$-especial-16-05-20161.pdf خ. Consulta: 7 de junio de 2017. 
destrezas y capacidades operativas para ejecutar políticas y fortalecer las capacidades de afrontamiento, con el fin de reducir el impacto adverso de las amenazas naturales y la posibilidad de que ocurra un desastre". ${ }^{4}$ Bajo ese concepto, este artículo tiene como objetivo analizar el marco jurídico ecuatoriano relativo a la gestión de riesgos antes y después del 16A.

En primer lugar, se describe el marco jurídico constitucional, legal y reglamentario que existía previo al terremoto, y, en segundo lugar, se analiza el marco jurídico generado a partir de decretos expedidos como respuesta al terremoto ocurrido en 2016. A partir de este análisis se concluye que las acciones de respuesta ante el 16A no se adecuaron plenamente al marco jurídico existente; al contrario, se presentó una sobreposición de funciones e instituciones. En consecuencia, el sistema de gestión de riesgos ecuatoriano no utilizó todas las normas jurídicas para reducir el impacto adverso del terremoto.

Por lo tanto, teniendo presente la obligación constitucional de proteger a las personas, las colectividades y la naturaleza de los efectos negativos de los desastres, el Ecuador requiere una ley de gestión de riesgos que adecue la dispersión de normas e instituciones, llene las lagunas jurídicas, y resuelva la inconstitucionalidad por no adecuación legislativa.

\section{MARCO JURÍDICO QUE REGULA(BA) LA GESTIÓN DE RIESGO DE DESASTRES (ANTES DEL 16 A)}

La Constitución del Ecuador establece normas jurídicas relacionadas con la gestión de riesgo de desastres. En primer lugar, señala que las personas víctimas de desastres, naturales o antrópicos, son parte de los grupos de atención prioritaria. ${ }^{5}$ Adicionalmente, reconoce la obligación del Estado de proteger "a las personas, las colectividades y la naturaleza frente a los efectos negativos de los desastres de origen natural o antrópico". ${ }^{6}$ La protección de las personas se debe realizar por medio de "la prevención ante el riesgo, la mitigación de desastres, la recuperación y mejoramiento de las condiciones sociales, económicas y ambientales, con el objetivo de minimizar la

4. Estrategia Internacional para la Reducción de Riesgo de Desastres de las Naciones Unidas (UNISDR) (Ginebra, 2009), 19. Disponible en 〈http://www.unisdr.org/files/7817_UNISDRTerminologySpanish.pdf〉. Consulta: 7 de junio de 2017.

5. Ecuador, Constitución de la República del Ecuador, Registro Oficial 449, 20 de octubre de 2008, art. 35.

6. Ibíd., art. 389. 
condición de vulnerabilidad". ${ }^{7}$ A continuación se detalla el marco jurídico relativo a la institucionalidad de la gestión de riesgos a nivel constitucional, legal y reglamentario.

La Constitución ecuatoriana señala que la gestión de riesgos de desastres será una competencia que actuará bajo el principio de descentralización subsidiaria, es decir que los Gobiernos Autónomos Descentralizados (GAD) Municipales son responsables directos dentro de su ámbito geográfico, pero cuando sus capacidades sean insuficientes, las instancias de mayor ámbito territorial y capacidad (GAD Provincial o Gobierno Nacional) brindarán el apoyo necesario, sin relevar de su responsabilidad a las instancias menores. ${ }^{8}$ Dentro de este sistema, el Estado central "ejercerá la rectoría a través del organismo técnico establecido en la ley". ${ }^{9}$

Este organismo técnico es responsable de articular las instituciones para que coordinen acciones a fin de:

a) Prevenir y mitigar los riesgos, así como enfrentarlos, recuperar y mejorar las condiciones anteriores a la ocurrencia de una emergencia o desastre", ${ }^{10}$

b) Realizar y coordinar las acciones necesarias para reducir vulnerabilidades y prevenir, mitigar, atender y recuperar eventuales efectos negativos derivados de desastres o emergencias en el territorio nacional. ${ }^{11}$

Es decir, es responsable, entre otros, de ejecutar y coordinar las acciones en las fases de respuesta, recuperación y rehabilitación ante desastres y emergencias, además de garantizar el financiamiento para el funcionamiento del Sistema, y coordinar la cooperación internacional en materia de gestión de riesgo. ${ }^{12}$

En el momento de la ocurrencia del terremoto del 16 de abril, la gestión de riesgo de desastres estaba regulada principalmente por dos cuerpos normativos con rango de ley: a) la Ley de Seguridad Pública y del Estado (en adelante Ley de Seguridad Pública); y b) el Código Orgánico de Organización Territorial, Autonomía y Descentralización (COOTAD) ${ }^{13}$ La primera incluye a la gestión de riesgos como parte del Sistema de Seguridad Pública y del Estado; sin embargo, no se regula el Sistema Nacional Descentralizado de Gestión de Riesgos. Además, la referida ley señala que la

7. Ibíd.

8. Ibíd., art. 390 .

9. Ibíd., art. 389.

10. Ibíd., art. 389.5.

11. Ibíd., art. 389.6.

12. Ibíd., art. 389.7.

13. Adicionalmente ver: Código Orgánico de Planificación y Finanzas Públicas de 2010, art. 64. ("En el diseño e implementación de los programas y proyectos de inversión pública, se promoverá la incorporación de acciones favorables al ecosistema, mitigación, adaptación al cambio climático y a la gestión de vulnerabilidades y riesgos antrópicos y naturales. [...]"). 
Secretaría de Gestión de Riesgos (SGR) ejercerá la rectoría de la gestión de riesgos. ${ }^{14}$ Sin embargo, la propia ley restringe las competencias constitucionales del organismo técnico; enfocándose solamente en la prevención, la reducción y la mitigación de riesgos y vulnerabilidades. Es decir, la Ley de Seguridad Pública no otorga a la SGR las competencias en relación a la respuesta, la recuperación y rehabilitación. De hecho, en el capítulo II "De los casos de estado de excepción", la Ley de Seguridad Pública señala que, en caso de desastre natural:

la planificación, organización, ejecución y coordinación de las tareas de prevención, rescate, remediación, asistencia y auxilio estarán a cargo del organismo responsable de la defensa civil, bajo la supervisión y control del Ministerio Coordinador de Seguridad o quien haga sus veces [...] El organismo responsable de la defensa civil actuará en coordinación con los gobiernos autónomos descentralizados y la sociedad civil, también contará con el apoyo de las Fuerzas Armadas y otros organismos necesarios para la prevención y protección de la seguridad, ejecutará las medidas de prevención y mitigación necesarias para afrontarlos y minimizar su impacto en la población. ${ }^{15}$

De la lectura de la Constitución y la Ley de Seguridad Pública, cabe preguntarse: primero, ¿es la Ley de Seguridad Pública la ley que establece el organismo técnico de gestión de riesgo? Si la respuesta es afirmativa, la segunda pregunta sería: ¿cuál es el organismo técnico que ejerce la rectoría? De conformidad con el artículo 11 podría ser la SGR; sin embargo, según el artículo 34, podría ser el organismo responsable de la defensa civil o el Ministerio Coordinador de Seguridad (MCS) o quien haga sus veces. ${ }^{16}$

Cabe mencionar que, mediante decreto ejecutivo y no por ley, se creó la SGR y esta institución asumió las funciones de la defensa civil, ${ }^{17}$ por lo que las responsa-

14. Ecuador, Ley de Seguridad Publica y del Estado (2009), art. 11.

15. Ibíd., art. 34.

16. Mediante el Decreto Ejecutivo No. 7 de 6 de junio de 2017 se suprimió el Ministerio Coordinador de Seguridad; sin embargo, no existe una mención específica sobre la transferencia de las responsabilidades en materia de gestión de riesgos; la única referencia es al equipo del Ministerio Coordinador de Seguridad encargado de la Secretaría Técnica del Consejo de Seguridad Pública y del Estado, donde el presidente tiene un plazo de 90 días para definir la instancia que asume referida responsabilidad, mientras tanto el referido equipo técnico pasará a formar parte del Secretaría de la Presidencia.

17. Mediante Decreto Ejecutivo No. 1046-A de 26 de abril de 2008, publicado en Registro Oficial No. 345 de 26 de mayo de 2008, se reorganiza la Dirección Nacional de Defensa Civil, mediante la figura de la Secretaría Técnica de Gestión de Riesgos adscrita al Ministerio de Coordinación de Seguridad Interna y Externa. Mediante Decreto Ejecutivo No. 42 del 10 de septiembre de 2009, publicado en el Registro Oficial No. 31 de 22 de septiembre de 2009, la Secretaría Técnica de Gestión de Riesgos pasará a denominarse Secretaría Nacional de Gestión de Riesgos. Mediante Decreto Ejecutivo No. 62, de 5 de agosto de 2013, la Secretaría Nacional de Gestión de Riesgos pasa a denominarse Secretaría de Gestión de Riesgos. 
bilidades en gestión de riesgos estarían divididas entre estas dos instituciones: SGR y MCS. Una tercera interrogante, ¿exclusivamente, cuando se declare un estado de excepción, el organismo responsable de la defensa civil es el competente en la respuesta a desastres, bajo supervisión y control del MCS? En consecuencia, ¿quién es el competente cuando no se ha declarado un estado de excepción? Como se señaló anteriormente, por el principio de descentralización subsidiaria, las instituciones locales serían las responsables dentro de su ámbito geográfico; y, para que intervenga el organismo de la defensa civil se requeriría de un estado de excepción. Finalmente, la Ley de Seguridad Pública tiene un enfoque exclusivamente antrópico de la gestión de riesgos, cuando la Constitución menciona que se debe proteger tanto a las personas como a las colectividades y la naturaleza.

El problema sobre la falta de claridad en la institucionalidad se repite en el COO$\mathrm{TAD}$, que señala que la competencia en materia de gestión de riesgos se "gestionará de manera concurrente y de forma articulada por todos los niveles de gobierno de acuerdo con las políticas y los planes emitidos por el organismo nacional responsable". ${ }^{18}$ Nuevamente, cabe la pregunta ¿cuál es el organismo nacional responsable, la SGR o el MCS?

Como corolario de la poca claridad sobre lo institucional en la legislación secundaria, y pese a que la Constitución señala que la gestión de riesgos se debe regular por medio de una ley, el Reglamento a la Ley de Seguridad Pública señala expresamente que "la [SGR] es el órgano rector y ejecutor del Sistema Nacional Descentralizado de Gestión de Riesgos". ${ }^{19}$ Sin embargo, la formulación de políticas, estrategias, planes y normas del Sistema Nacional Descentralizado de Gestión de Riesgos, se realizará "bajo la supervisión del [MCS], para la aprobación del Presidente de la República". ${ }^{20}$ Este reglamento también establece las competencias para la SGR. En términos generales, el reglamento repite las competencias constitucionales asignadas para el ente rector de la gestión de riesgos; sin embargo, añadió las siguientes competencias:

1. Diseñar programas de educación, capacitación y difusión orientados al fortalecimiento de las capacidades de las instituciones y los ciudadanos en la gestión de riesgos;

2. Coordinar la cooperación entre la ayuda humanitaria y la información para hacer frente a situaciones emergentes y / o desastres causados por fenómenos naturales, socio-naturales o antropogénicos a nivel nacional e internacional. ${ }^{21}$

18 Ecuador, Código Orgánico de Organización Territorial, Autonomía y Descentralización (2010), art. 140.

19. Reglamento a la Ley de Seguridad Pública y del Estado de 2010, artículo 3. El artículo 18 reitera que el Estado ejerce la rectoría del Sistema Nacional Descentralizado de Gestión de Riesgos a través de la SGR.

20. Ibíd., artículo 18.b.

21. Ibíd., artículo 3.g-h. 
Asimismo, se modificó la responsabilidad relacionada con la financiación, ya que la Constitución dice que "garantizar una financiación suficiente y oportuna", ${ }^{22}$ mientras que el reglamento establece "gestionar los fondos necesarios". ${ }^{23}$ El reglamento institucionaliza y organiza el Sistema Descentralizado de Gestión de Riesgos, ${ }^{24}$ que "incluye el conjunto de actividades de prevención, mitigación, preparación, alerta, respuesta, rehabilitación y reconstrucción de los efectos de los desastres". ${ }^{25}$

Además, el referido reglamento establece el Comité Consultivo Nacional de Gestión de Riesgos, el cual es presidido por la SGR e integrado por todos los ministerios coordinadores. ${ }^{26}$ Paralelamente, el Reglamento señala que los Comités de Operaciones de Emergencia (COE) "son instancias interinstitucionales responsables en su territorio de coordinar las acciones tendientes a la reducción de riesgos, y a la respuesta y recuperación en situaciones de emergencia y desastre". ${ }^{27}$ Bajo el principio constitucional de descentralización subsidiaria, los $\mathrm{COE}$ son responsabilidad directa de las instituciones dentro de su ámbito geográfico; es decir, existirán COE nacional, provinciales y cantonales.$^{28}$ El Reglamento señala que la SGR normará la conformación y funcionamiento de los COE. ${ }^{29}$ Bajo ese mandato, en el año 2014, la SGR adoptó el Manual de Gestión de Riesgos para Emergencias y Desastres. Este manual organizó las instituciones para todos los componentes de gestión de riesgos, incluyendo la respuesta a desastres o emergencias. ${ }^{30}$

Pese a que la Constitución del Ecuador dispone que mediante ley se debe establecer un órgano técnico rector de la gestión de riesgos, desde el año 2008 no se ha adoptado la Ley de Gestión del Riesgo de Desastres. ${ }^{31}$ En la actualidad, ciertas normas

22. CRE, artículo 389.7.

23. Reglamento a la Ley de Seguridad Pública y del Estado de 2010, artículo 3.e.

24. Ibíd., artículos 15-24.

25. Ibíd., artículos 16 .

26. En junio de 2017, todos los ministerios coordinadores fueron suprimidos, por lo que esta norma debe ser actualizada.

27. Reglamento a la Ley de Seguridad Pública y del Estado (2010), art. 24.

28. Ibíd.

29. Ibíd.

30. Secretaría de Gestión de Riesgos, Manual del Comité de Gestión de Riesgos, Resolución No. 38. Publicación del Registro Oficial, Edición Especial No. 211, 25 de noviembre de 2014.

31. Asamblea Nacional, Comisión Especial Permanente sobre Biodiversidad y Recursos Naturales, Proyecto de Ley de Cambio Climático, Informe para el Segundo Debate. (7 de mayo, 2013). Disponible en 〈http:// ppless.asambleanacional.gob.ec/alfresco/d/d/workspace/SpacesStore/a35d00fe-7214-4497-8eec-6e 7bdb89f28d/Informe\%20Segundo\%20Debate\%20Tr.\%20135907.pdf〉. Consulta: 7 de mayo de 2017.

(El 10 de julio de 2012, un legislador presentó el "Proyecto de Ley de Gestión de Riesgos del Sistema Nacional Descentralizado", sin embargo, el 7 de mayo de 2013 sobre el informe para el segundo debate, el proyecto fue rechazado y archivado). 
relacionadas con la gestión de riesgo de desastres están dispersas en diferentes leyes y reglamentos.

Bajo este confuso marco jurídico ocurrió un terremoto en la costa ecuatoriana el 16 de abril de 2016.

\section{RESPUESTA JURÍDICA E INSTITUCIONAL ANTE EL TERREMOTO DEL 16A}

La poca claridad existente en relación a la organización de la gestión de riesgo se agudizó luego del terremoto del 16A. Hasta junio de 2017 se han adoptado diez decretos de estado de excepción relacionados con el terremoto. Además, mediante decreto se creó también un comité de reconstrucción y reactivación de las zonas afectadas por el terremoto.

\section{ESTADOS DE EXCEPCIÓN POR EL 16A}

La Constitución menciona los desastres "naturales" como una causal para la declaratoria de estado de excepción, ${ }^{32}$ el cual es "un mecanismo o arreglo normativoconstitucional [...] para garantizar los derechos de los ciudadanos dentro del territorio nacional en caso de eventos imprevisibles, derechos que no pueden ser protegidos con los mecanismos jurídico-institucionales regulares". ${ }^{33}$

Por lo tanto, un decreto de estado de excepción "será dictado en caso de estricta necesidad, es decir, si el orden institucional no es capaz de responder a las amenazas de seguridad de las personas y del Estado". ${ }^{34}$ En este sentido, la Corte Constitucional, como el "máximo órgano de control, interpretación constitucional y de administración de justicia en esta materia", ${ }^{35}$ analizó la constitucionalidad de los Decretos Ejecutivos: a) 1001 del 17 de abril de 2016 por el cual se declaró el estado de excepción a causa de los eventos telúricos presentados el 16 de abril de 2016; y, b) 1002 del 18 de abril de 2016 mediante cual se amplió la extensión en el tiempo del primer decreto. Posteriormente se han dictado de manera periódica renovaciones del estado de excepción,

32. Ecuador, Constitución de la República del Ecuador, art. 164.

33. Corte Constitucional del Ecuador, dictamen No. 003-15-DEE-CC, caso No. 009-11-EE del 13 de mayo de 2015.

34. Ecuador, Ley de Seguridad Pública y del Estado (2010), art. 29.

35. Ecuador, Constitución de la República del Ecuador, art. 436. 
en los que se pueden observar como operaron ciertos aspectos institucionales y como se suspendieron ciertos derechos. ${ }^{36}$

En su parte considerativa, todos los decretos se fundamentan en el artículo 389 de la Constitución, el cual señala que el Estado ejercerá la rectoría de la gestión de riesgos mediante un organismo técnico establecido en la ley. ${ }^{37}$ De hecho, en los considerandos también se dice que la SGR es el organismo rector del referido sistema. ${ }^{38}$ Sin embargo, los propios decretos no tienen concordancia con la institucionalidad mencionada. Por citar un caso en concreto, el MCS solicitó en la mayoría de ocasiones la renovación del estado de excepción. ${ }^{39}$ Por su parte, la SGR solicitó la declaratoria de estado de excepción solamente en dos ocasiones. ${ }^{40}$ Estos hechos muestran la falta de institucionalidad en la respuesta al terremoto.

Las medidas adoptadas durante la excepción constituye otro punto donde se visibiliza esta falta de institucionalidad. Mediante los dos primeros decretos de estado de excepción se establecieron tres medidas no ordinarias:

a) Disponer la movilización en todo el territorio nacional de tal manera que todas las entidades de la administración pública central e institucional, en especial las Fuerzas Armadas y la Policía Nacional, y los gobiernos autónomos descentralizados de las provincias afectadas, coordinen esfuerzos con el fin de ejecutar las acciones necesarias e indispensables para mitigar y prevenir los riesgos, así como enfrentar, recuperar y mejorar las condiciones adversas [...]

b) Se ordena al Ministerio de Finanzas que sitúe los fondos públicos necesarios para atender la situación de excepción, pudiendo utilizar todas las asignaciones presupuestarias disponibles, salvo las destinadas a salud y educación.

36. Mediante los decretos ejecutivos No. 1101 de 16 de junio de 2016, No. 1116 de 15 de julio de 2016, No. 1191 de 13 de septiembre de 2016, No. 1215 de 14 de octubre de 2016, No. 1274 de 13 de diciembre de 2016, No. 1295 de 12 de enero de 2017, No. 1338 de 12 de marzo de 2017, No. 1364 de 12 de abril de 2017 y No. 27 de 12 de junio de 2017.

37. Decreto Ejecutivo No. 1001 del 17 de abril de 2016, considerando cuarto.

38. Ibíd., considerando quinto. Decreto Ejecutivo No. 1101 de 16 de junio de 2016, considerandos cuatro, cinco y seis.

39. Decretos ejecutivos: 1101 de 16 de junio de 2016, considerando diez; 1191 de 13 de septiembre de 2016, considerando nueve; 1215 de 14 de octubre de 2016, considerando nueve; 1279 de 14 de diciembre de 2016, considerando nueve; 1295 de 12 de enero de 2017, considerando diez; 1364 de 12 de abril de 2017, considerando diez; y, 1338 de 12 de marzo de 2017, considerando nueve.

40. Decretos ejecutivos: 1116 de 15 de julio de 2016, considerando nueve; y, 27 de 13 de junio de 2017, considerando nueve. 
c) Se dispone las requisiciones a las que haya lugar para solventar la emergencia producida, las mismas que se harán en casos de extrema necesidad y en estricto cumplimiento del ordenamiento jurídico. ${ }^{41}$

Dentro de estas medidas, llama la atención la movilización de todas las entidades de la administración pública, sin un orden ni un liderazgo específico del organismo rector. De hecho, el Presidente de la República encarga la ejecución de los decretos de estado de excepción a los Ministerios: Coordinador de Seguridad, Interior, Defensa, Finanzas, Salud, Inclusión Económica y Social; y la SGR. ${ }^{42}$ Es decir la implementación de las medidas excepcionales está a cargo de diversas instituciones, sin establecer la rectoría de la SGR o del MCS.

Adicionalmente, a partir de la renovación del estado de excepción, se agrega la suspensión de los siguientes derechos:

el ejercicio del derecho a la inviolabilidad de domicilio y de libre tránsito de los afectados por el terremoto [...], en las provincias de Manabí y Esmeraldas, por cuanto algunos ciudadanos pretenden retornar a sus hogares situados en inmuebles que precisamente constituyen un riesgo para su vida o integridad física. El [MCS] determinará la forma de aplicar esta medida para conseguir la finalidad señalada. ${ }^{43}$

La propia redacción del artículo indica que de facto estos derechos ya se encontraban suspendidos porque luego del terremoto algunas personas pretendían ingresar a lugares donde su vida e integridad física se encontraban en riesgo. En efecto, durante los primeros días de la respuesta sí se suspendieron varios derechos de rango constitucional como la inviolabilidad del domicilio por el necesario ingreso de equipos de búsqueda y rescate en varias viviendas; además, se limitó el derecho a la libre movilidad, ya que se prohibió el ingreso a ciertas zonas de riesgo. Por ejemplo, en mayo, el MCS informó que para ingresar a la zona cero de Portoviejo se requería un salvoconducto. ${ }^{44}$

41. Corte Constitucional del Ecuador, dictamen No. 002-16-DEE-CC, caso No. 002-16-EE del 27 de abril de 2017, 13.

42. Decreto ejecutivo No. 1001 del 17 de abril de 2016, artículo 6. En los decretos en que se renueva el estado de excepción se encarga la ejecución a los mismos ministerios; salvo el último decreto, No. 27, en el cual ya no se menciona al MCS debido a que fue suprimido.

43. Decretos ejecutivos: 1101 de 16 de junio de 2016, artículo 3; y, 1116 de 15 de julio de 2016, art. 3.

44. Ministerio Coordinador de Seguridad, Boletín de Prensa No. 101, "Gobierno Autónomo Descentralizado emitirá salvoconductos para ingreso a la Zona Cero", 23 de mayo de 2016. Disponible en «http://www. seguridad.gob.ec/gobierno-autonomo-descentralizado-emitira-salvoconductos-para-ingreso-a-la-zonacero/s. Consulta: 7 de mayo de 2017. 
Consecuentemente, llama la atención que se suspendan estos derechos luego de dos meses de ocurrido el terremoto. Adicionalmente, un aspecto institucional que salta a la vista es que se encarga al MCS determinar cómo aplicar la suspensión de derechos. Este punto se complejiza en el decreto No. 27, que, luego de la supresión del MCS, señala que "los ministerios del Interior y de Defensa determinarán la forma de aplicar la suspensión de derechos". ${ }^{45}$ Se debe recordar que al ente rector de la gestión de riesgos le correspondería "realizar y coordinar las acciones necesarias para reducir vulnerabilidades y prevenir [...] eventuales efectos negativos derivados de desastres" ${ }^{46}$ Por lo que el organismo rector debería determinar la forma de aplicar la suspensión de derechos en el contexto del desastre.

Por otro lado, dentro de los dictámenes previos de la Corte Constitucional se observan varios aspectos que evidencian la falta de institucionalidad en la gestión de riesgo. En primer lugar, se observa que la Corte Constitucional notificó de los casos de manera indistinta a ciertas instituciones. En el caso número 0002-16-EE, la jueza sustanciadora del proceso constitucional notificó al Presidente de la República, la Presidencia de la Función Legislativa, los ministerios: Coordinador de Seguridad, Interior, Defensa, Finanzas, Salud, Inclusión Económica y Social, la SGR; y, la Procuraduría General del Estado (PGE). De la misma manera, sobre los decretos 1191 y 1295 se notificó a las mismas instituciones ${ }^{47}$ Sin embargo, del decreto No. 1101, el juez sustanciador notificó al Presidente de la República, la PGE y la Presidencia de la Asamblea Nacional; ${ }^{48}$ de los decretos 1116, 1215 y 1364 se notificó exclusivamente al Presidente de la República; ${ }^{49}$ y, del decreto 1274 no se notificó a persona o institución alguna.

Adicionalmente, cuando la Corte Constitucional debía evaluar la idoneidad de las medidas, solo se limitó a mencionar su "fin legítimo", es decir, la mitigación de los hechos y la garantía de la seguridad e integridad de las personas. ${ }^{50}$ Para valorar la idoneidad de las medidas, la Corte Constitucional debería emplear herramientas sobre la gestión de riesgos de desastre, incluyendo los informes del organismo rector. ${ }^{51}$

45. Decreto ejecutivo No. 27 de 12 de junio de 2017.

46. Ecuador, Constitución de la República del Ecuador, art. 329.6.

47. Corte Constitucional del Ecuador, dictamen No. 008-16-DEE-CC, caso No. 005-16-EE del 29 de noviembre de 2016, 1. Corte Constitucional del Ecuador, dictamen No. 002-17-DEE-CC, caso No. 002-17-EE del 8 de febrero de $2017,1$.

48. Ibíd., dictamen No. 004-16-DEE-CC, caso No. 003-16-EE del 3 de agosto de 2016, 1.

49. Ibíd., dictamen No. 005-16-DEE-CC, caso No. 004-16-EE del 3 de agosto de 2016, 1; dictamen No. 007-16-DEE-CC, caso No. 006-16-EE del 22 de noviembre de 2016, 1; dictamen No. 006-17-DEE-CC, caso No. 004-17-EE del 19 de mayo de 2017, 2.

50. Ibíd., dictamen No. 002-16-DEE-CC, caso No. 002-16-EE del 27 de abril de 2017, 17.

51. Ecuador, Constitución de la República del Ecuador, art. 389.1. 
Finalmente, en todos los casos, al concluir su análisis jurídico y bajo un formato similar, la Corte Constitucional emitió un dictamen favorable de constitucionalidad a la declaratoria de estado de excepción. Como señala Juan Pablo Aguilar, la Corte Constitucional "a la hora de examinar cada decreto [de estado de excepción] recurre a cualquier pirueta mental para conseguir que, a la larga, se justifique precisamente la clase de decisiones que la teoría busca descalificar". ${ }^{52}$ Por ejemplo, justifica la declaratoria de estado de excepción por la "necesidad de articular las instituciones para que coordinen acciones y esfuerzos a fin de prevenir y mitigar los riesgos; así como para enfrentarlos, recuperar y mejorar las condiciones adversas que provoquen los eventos telúricos" ${ }^{53}$ Sin embargo, textualmente esa es una de las funciones constitucionales del organismo técnico de la gestión de riesgo. ${ }^{54}$ De igual manera, la Corte Constitucional dijo que para emprender la recuperación y reconstrucción no es suficiente el marco jurídico ordinario; por lo que se requiere la declaratoria de estado de excepción para hacer efectiva la intervención del Estado y sus instituciones en el proceso de reconstrucción. ${ }^{55} \mathrm{Sin}$ embargo, los decretos de estado de excepción no fueron el único instrumento jurídico para regular la respuesta institucional ante el terremoto, dado que mediante decreto ejecutivo se instituyó un comité para la reconstrucción.

\section{Del Comité de Reconstrucción y Reactivación}

Sumando a esta dispersión de normas e instituciones, mediante Decreto Ejecutivo No. 1004 del 26 de abril de 2016, el Presidente de la República creó el Comité para la Reconstrucción y Reactivación Productiva. En el decreto se considera que para implementar políticas, proyectos y programas para la reconstrucción y reactivación, es necesaria una institucionalidad que establezca mecanismos de coordinación transversal. ${ }^{56}$ En este sentido, los objetivos del Comité de reconstrucción son:

a) Ejecutar la construcción y reconstrucción de infraestructura necesaria para mitigar los efectos del terremoto; $y$,

52. Juan Pablo Aguilar, "Entre la retórica de lo nuevo y la persistencia del pasado: La Corte Constitucional y los estados de excepción”, Iuris Dictio, No. 13 (2010): 65.

53. Corte Constitucional del Ecuador, dictamen No. 005-16-DEE-CC, caso No. 004-16-EE del 3 de agosto de 2016, 5.

54. Ecuador, Constitución de la República del Ecuador, art. 389.5.

55. Corte Constitucional del Ecuador, dictamen No. 004-16-DEE-CC, caso No. 003-16-EE del 3 de agosto de 2016, 13.

56. Decreto ejecutivo No. 1004 del 26 de abril de 2016, considerandos quintos y sexto. 
b) Implementar planes, programas, acciones y políticas públicas para la reactivación de la producción y de empleo en las zonas afectadas. ${ }^{57}$

Este comité es presidido por el Vicepresidente de la República; además, lo integran: el Secretario Nacional de Planificación y Desarrollo; el Ministro Coordinador de Desarrollo Social; el Ministro Coordinador de Producción, Empleo y Competitividad; el Ministro Coordinador de Seguridad; el Prefecto de Manabí; y dos alcaldes en representación de las zonas afectadas. ${ }^{58}$

En primer lugar, se observa la ausencia de la SGR, supuesto organismo rector de la gestión de riesgos. Es más, el propio decreto 1004, en los considerandos, cita el artículo 389 de la Constitución, específicamente cita varias de las funciones principales del organismo técnico de la gestión de riesgos, relacionadas con: a) el fortalecimiento de capacidades para identificar y reducir riesgos; b) la articulación de instituciones para que coordinen acciones de prevención y mitigación de riesgos, así como para enfrentarlos, recuperar y mejorar las condiciones anteriores a la ocurrencia de una emergencia o desastre; c) la reducción de vulnerabilidades y la prevención, mitigación, atención y recuperación ante efectos negativos derivados de desastres o emergencias; y d) el financiamiento para el funcionamiento del Sistema de gestión de riegos, contemplando la coordinación de la cooperación internacional dirigida a la gestión de riesgo. ${ }^{59}$ Específicamente, el Comité de Reconstrucción ejecuta acciones en las siguientes etapas posdesastre:

- Emergencia: rescate, salud, alimentación, albergues, remoción de escombros y demolición de edificaciones inhabilitadas. El [MCS] es responsable de este eje.

- Reconstrucción: construcción y reconstrucción de infraestructura pública, rehabilitación integral de servicios públicos, diseño, planificación y construcción de vivienda para damnificados. Cada Ministerio rector es responsable de este eje.

- Reactivación productiva: ejecución de planes, programas, políticas y regulaciones productivas, reactivación de empleo local y nacional; y, de financiamiento para las zonas afectadas. El Ministerio de Coordinación de la Producción, Empleo y Competitividad es el responsable de este eje. ${ }^{60}$

Evidentemente, el Comité de Reconstrucción asumió competencias que le correspondían al organismo rector de la gestión de riesgos de desastres. Adicionalmente, a

\footnotetext{
57. Ibíd., art. 1.

58. Ibíd., art. 2 .

59. Ecuador, Constitución de la República del Ecuador, art. 389, numerales 4, 5, 6 y 7. Citados en Decreto ejecutivo No. 1004 del 26 de abril de 2016, considerando segundo.

60. Decreto ejecutivo No. 1004 del 26 de abril de 2016, art. 5.
} 
nivel institucional, se creó la Secretaría Técnica del Comité para la Reconstrucción y Reactivación Productiva, con el objetivo de coordinar y dar seguimiento a los ejes de acción del Comité de Reconstrucción y Reactivación Productiva.$^{61}$ Por otro lado, se reformaron las funciones de la Empresa Pública de Desarrollo Estratégico Ecuador Estratégico, incluyendo la siguiente:

Planificar, diseñar, evaluar, priorizar, financiar y ejecutar los planes, programas y proyectos de inversión necesarios para la construcción y reconstrucción de infraestructura pública y vivienda y de reactivación productiva y de empleo en las zonas de afectación de desastres naturales y de influencia de los proyectos de sectores estratégicos. ${ }^{62}$

Finalmente, se dispuso que el Ministerio de Finanzas cree una cuenta específica, alimentada por recursos internos y externos, para la construcción, reconstrucción y reactivación productiva de las zonas afectadas por el terremoto, ${ }^{63}$ lo cual muestra la dispersión de instituciones en la respuesta al desastre.

\section{CONCLUSIONES}

Antes del terremoto del 16 de abril de 2016, y a pesar del mandato constitucional, el marco jurídico de la gestión de riesgo de desastres era disperso y poco claro respecto a la institucionalidad. La Ley de Seguridad Pública y el COOTAD incluyen normas sobre la materia; sin embargo, las mismas no cumplen con el espíritu constituyente, dado que no cubren todas las fases de la gestión de riesgo de desastres.

A nivel institucional, la Ley de Seguridad Pública otorga funciones en materia de gestión de riesgos a la SGR, el MCS y la Defensa Civil. Sin embargo, el reglamento a la Ley de Seguridad Pública otorga competencias exclusivamente a la SGR. El problema radica en que las tres instituciones en referencia han sido creadas, reformadas y suprimidas por decretos ejecutivos y no por ley, como ordena la Constitución en su artículo 389. Referido reglamento además agrega competencias y modifica otras, en contraposición a las disposiciones constitucionales y legales.

En la respuesta al terremoto, la gobernanza de la gestión del riesgo fue aún más confusa. Diversas instituciones fueron designadas para la implementación de los estados de excepción, pero otras fueron encargadas de la reconstrucción. En ambos

61. Ibíd., art. 6. Ver también: Acuerdo Vicepresidencial No. 001-2013 de 24 de mayo de 2013 (Estatuto orgánico por procesos de la Vicepresidencia de la República, capítulo V).

62. Decreto Ejecutivo No. 1004 del 26 de abril de 2016, art. 8.

63. Ibíd., art. 9. 
casos se pasó por alto al supuesto organismo técnico rector de la gestión de riesgo. Asimismo, el MCS y la SGR solicitaron de manera indistinta la renovación del estado de excepción, por lo que surge la pregunta ¿quién debía requerir la renovación del estado de excepción? Bajo la norma constitucional debería ser el organismo técnico de la gestión de riesgos, establecido por ley. Tampoco existió claridad sobre cuál era la institución encargada de aplicar la suspensión de derechos.

En materia de gestión de riesgos actualmente existe una inconstitucionalidad por omisión legislativa. ${ }^{64}$ La declaratoria de inconstitucionalidad por omisión consiste en evidenciar que las violaciones a derechos o las afectaciones a la observancia a las normas constitucionales pueden darse por una omisión de un mandato constitucional que no ha sido atendido; por ejemplo, cuando no se atiende condiciones específicas exigidas por la Constitución. ${ }^{65}$ En el presente análisis se ha demostrado que en materia de gestión de riesgos la función legislativa no ha establecido, mediante ley, el organismo rector de la gestión de riesgos, por lo que la designación ha quedado al albedrío de la función ejecutiva.

Con el fin de profundizar el estudio de la gobernanza de la gestión del riesgo durante el terremoto sería oportuno analizar los informes del Comité de Reconstrucción, de la Contraloría General del Estados y del Sistema de Contratación Pública.

Luego del terremoto se han adoptado nuevas leyes como la Ley Orgánica de Ordenamiento Territorial, Uso y Gestión del Suelo, y, el Código Orgánico del Ambiente, que contienen disposiciones relacionadas con la gestión de riesgo de desastres, por lo que su institucionalidad puede haber sido, nuevamente, modificada.

Ante esta dispersión de normas e instituciones, la función legislativa debe adoptar una ley de gestión de riesgos que adecue esa dispersión, llene las lagunas jurídicas, y solucione la inconstitucionalidad por no adecuación legislativa.

\section{BIBLIOGRAFÍA}

Acuerdo Vicepresidencial No. 001-2013 de 24 de mayo de 2013 (Estatuto orgánico por procesos de la Vicepresidencia de la República, capítulo V).

Aguilar, Juan Pablo. "Entre la retórica de lo nuevo y la persistencia del pasado: La Corte Constitucional y los estados de excepción”. Iuris Dictio, No. 13 (2010).

64. Ecuador, Constitución de la República del Ecuador, art. 424, 425 y 426. Ley Orgánica de Garantías Jurisdiccionales y Control Constitucional de 2009, art. 128-130.

65. Paúl Córdova, Derecho Procesal Constitucional (Quito: Corporación de Estudios y Publicaciones, 2016), 254-60. 
Asamblea General de Naciones Unidas. Resolución A/RES/69/283: Marco de Sendai para la Reducción del Riesgo de Desastres 2015-2030.

Asamblea Nacional, Comisión Especial Permanente sobre Biodiversidad y Recursos Naturales. Proyecto de Ley de Cambio Climático, Informe para el Segundo Debate (2013).

Córdova, Paúl. Derecho Procesal Constitucional. Quito: Corporación de Estudios y Publicaciones, 2016.

Corte Constitucional del Ecuador. Dictamen No. 003-15-DEE-CC, caso No. 009-11-EE del 13 de mayo de 2015.

—. Dictamen No. 002-16-DEE-CC, caso No. 002-16-EE del 27 de abril de 2017.

-. Dictamen No. 004-16-DEE-CC, caso No. 003-16-EE del 3 de agosto de 2016.

—. Dictamen No. 005-16-DEE-CC, caso No. 004-16-EE del 3 de agosto de 2016.

—. Dictamen No. 007-16-DEE-CC, caso No. 006-16-EE del 22 de noviembre de 2016.

—. Dictamen No. 008-16-DEE-CC, caso No. 005-16-EE del 29 de noviembre de 2016.

—. Dictamen No. 001-17-DEE-CC, caso No. 008-16-EE del 25 de enero de 2017.

-. Dictamen No. 002-17-DEE-CC, caso No. 002-17-EE del 8 de febrero de 2017.

—. Dictamen No. 005-17-DEE-CC, caso No. 003-17-EE del 29 de marzo de 2017.

—. Dictamen No. 006-17-DEE-CC, caso No. 004-17-EE del 19 de mayo de 2017.

Estrategia Internacional para la Reducción de Riesgo de Desastres de las Naciones Unidas (UNISDR), Ginebra, 2009.

Ministerio Coordinador de Seguridad. Boletín de Prensa No. 101, “Gobierno Autónomo Descentralizado emitirá salvoconductos para ingreso a la Zona Cero”, 23 de mayo de 2016.

Secretaría de Gestión de Riesgos. "Informe de Situación, No. 65 (2016).

—. "Informe de Situación No. 71 (2016).

-. Manual del Comité de Gestión de Riesgos. Resolución No. 38. Publicación del Registro Oficial, Edición Especial No. 211, 25 de noviembre de 2014. 